\title{
Transradial Carotid Artery Stenting in a Patient with Leriche Syndrome
}

\section{Sung Ho Kim, Dong Gyu Yeo, Gwang Su Lee}

Department of Neurosurgery, Soonchunhyang University Gumi Hospital, Gumi, Korea

\begin{abstract}
We report a case of the transradial approach for carotid artery stenting (CAS) as a useful alternative to the traditional transfemoral approach when femoral access is not available. A 50-year-old male visited our emergency room with dysarthria and right-side weakness. Magnetic resonance imaging showed acute cerebral infarction on the left middle cerebral artery territory and carotid stenosis on both sides. CAS for each carotid stenosis was attempted by the usual femoral access, but there was no pulse on either side of the femoral artery, and computed tomography angiogram of the lower extremity revealed Leriche syndrome, which is an aortoiliac occlusive disease. So, we changed the access to the radial artery and was successfully performed.
\end{abstract}

Keywords: Transradial approach; Carotid artery stenting; Aortoiliac occlusive disease

\section{INTRODUCTION}

Carotid artery stenting (CAS) is an effective alternative to carotid endarterectomy (CEA) for the treatment of carotid stenosis. The transfemoral approach is the traditional approach in performing CAS with a high success rate. However, there are situations a transfemoral approach can not be done such as extensive atherosclerotic disease in the aortic arch, iliofemoral occlusive disease, dissection of the thoracic aorta, etc. In such a case, the transradial approach can be a useful alternative approach. We report a case of symptomatic carotid artery stenosis combined with Leriche syndrome which is an aortoiliac occlusive disease, treated by CAS by transradial approach with good result.

\section{CASE REPORT}

A 50-year-old male visited the emergency room of Soonchunhyang University Gumi Hospital with dysarthria and right-side weakness. The patient had a 45 pack-year smoking history and no other underlying disease. His National Institutes of Health Stroke Scale score was 3. Brain magnetic resonance imaging (Fig. 1A) showed acute cerebral infarction on left middle cerebral artery territory and carotid computed tomography (CT) angiography (Fig. 1B) revealed bilateral carotid stenosis (left carotid stenosis: $50 \%$ stenosis, right carotid stenosis: 90\% based on NASCET [North American Symptomatic Carotid Endarterectomy Trial] criteria). Work up for detection of any other stroke risk factor was done, including the cardiologic evaluation and there was no other stroke risk factor besides carotid stenosis. First, the patient was on dual antiplatelet (aspirin $100 \mathrm{mg}$, clopidogrel $150 \mathrm{mg}$ ) medication, and hydration and neurological deficit subsided after 2 days of admission. The left carotid stenosis was symptomatic with $50 \%$ stenosis and the right carotid stenosis was asymptomatic but was severe, so both carotid stenoses were planned to be treated by CAS. The more severe right side was planned to be treated first and then the left side. Dual antiplatelets were given for about a month so no other antiplatelet loading was done. On procedure, both the femoral artery had no pulse so we could not proceed with the procedure. CT angiography of the lower extremity was done showing Leriche syndrome, an aortoiliac occlusive disease involving the blockage of the abdominal aorta as it transitions into the common iliac arteries (Fig. 2). It seemed difficult to perform CAS because the usual and familiar femoral access was not available, so we planned CEA. But the patient strongly insisted on CAS and we finally planned CAS through a radial approach. Allen's test was negative, and we punctured the right radial artery and the $5 \mathrm{~F}$ arterial sheath was inserted and then switched to $7 \mathrm{~F}$ and $8 \mathrm{~F}$ sheath sequentially not to rupture of the small radial artery by the $8 \mathrm{~F}$ sheath 

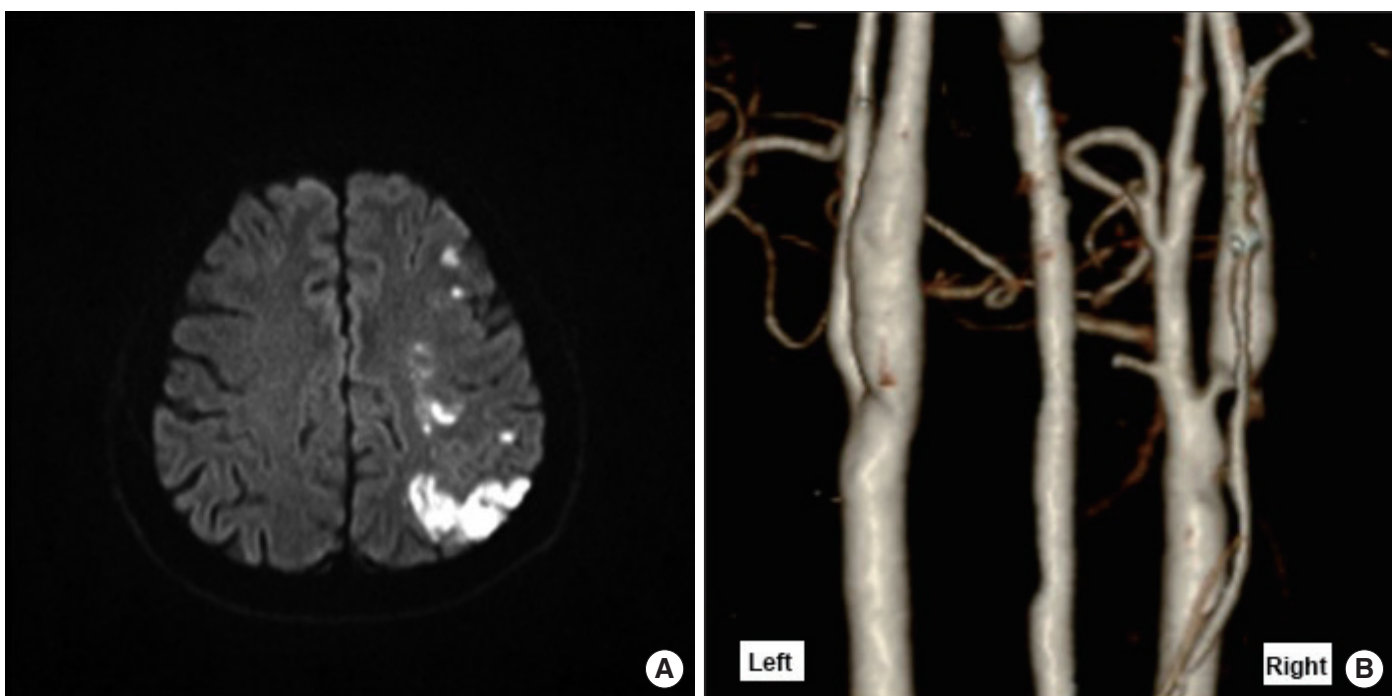

Fig. 1. A 50-year-old male visited our emergency room with dysarthria and right-side weakness. (A) Initial diffusion magnetic resonance imaging showed acute cerebral infarction on the left middle cerebral artery territory. (B) Carotid computed tomography angiography revealed bilateral carotid stenosis (left carotid stenosis: 50\% stenosis, right carotid stenosis: 90\% based on NASCET [North American Symptomatic Carotid Endarterectomy Trial] criteria).

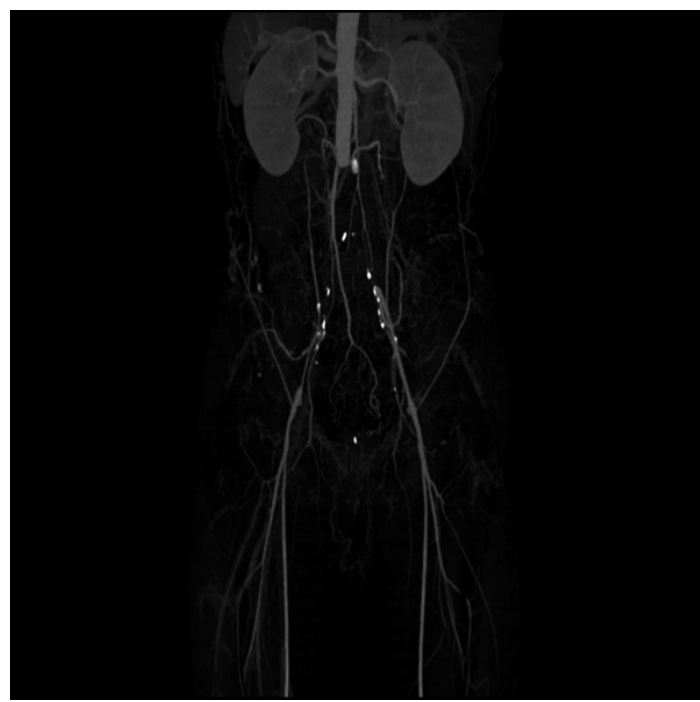

Fig. 2. Computed tomography angiography of the lower extremity was done showing Leriche syndrome, an aortoiliac occlusive disease involving the blockage of the abdominal aorta as it transitions into the common iliac arteries.

we planned to use (Fig. 3). Verapamil (2.5 mg) and nitroglycerine $(200 \mu \mathrm{g})$ were injected via arterial sheath in a radial artery to prevent arterial spasm, and 3,000 unit of heparin were given intravenously to match ACT of 200-300 seconds. There was an extreme angulation at the right common carotid artery (CCA) origin so Simmons catheter was used to cannulate the CCA and an angiogram was done (Fig. 4). It was difficult to advance the guiding catheter because of the extreme angulation between the brachiocephalic trunk and CCA. The guiding catheter was pushed to the

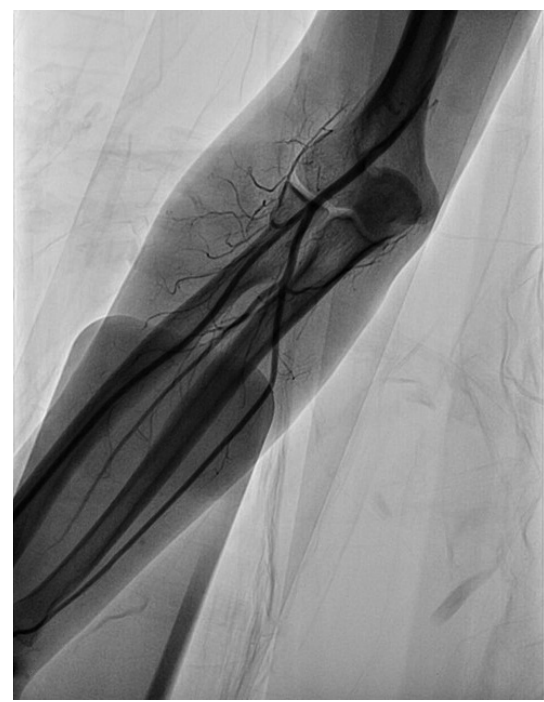

Fig. 3. The right radial artery was punctured and a $5 F$ arterial sheath was inserted and then switched to $7 \mathrm{~F}$ and $8 \mathrm{~F}$ sheath sequentially.

CCA over the wire, and failed so the buddy wire was attempted, and failed, and finally over Simmons catheter but failed. The VISTA BRITE TIP, 8F JL 6 (Cordis Corp., Santa Clara, CA, USA) guiding catheter which is used for coronary artery stenting had a shape appropriate for a selection of CCA so it was planned to be used. It had a ' $C$ ' shape so we reshaped the tip using hot steam to a 'U' shape, a similar shape with Simmons catheter and directly cannulated the CCA with the guiding catheter using the "pullback and rotate" technique, the same technique used for Simmons 


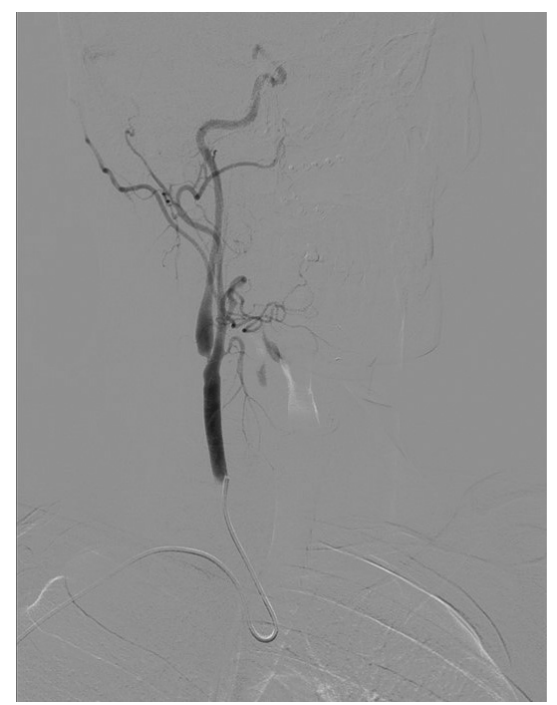

Fig. 4. Simmons catheter was used to cannulate the common carotid artery and an angiogram was done.

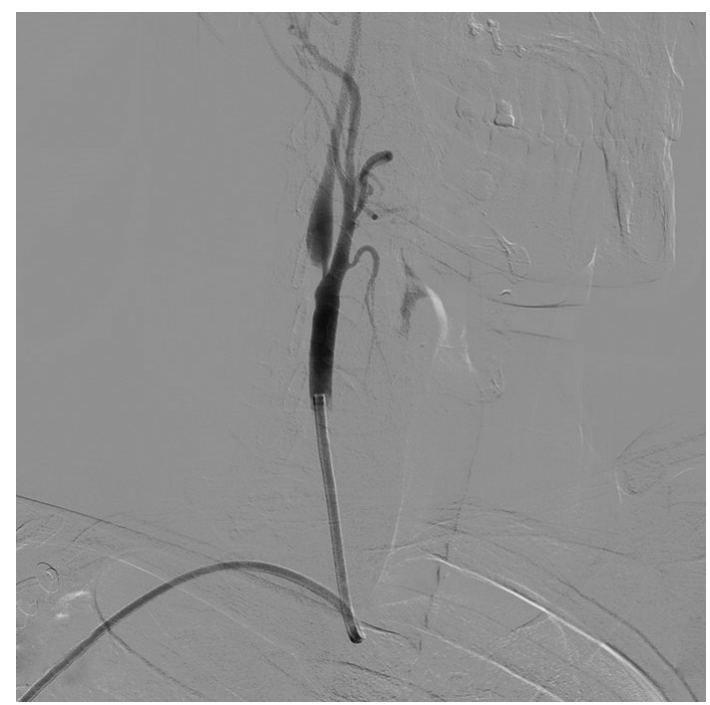

Fig. 5. The VISTA BRITE TIP, 8F JL 6 (Cordis Corp., Santa Clara, CA, USA) guiding catheter used for coronary artery stenting had a shape appropriate for the selection of CCA. It had a ' $\mathrm{C}$ ' shape so we reshaped the tip using hot steam to a 'U' shape, a similar shape with Simmons catheter, and directly cannulated the CCA with the guiding catheter using the "pullback and rotate" technique, the same technique used for Simmons catheter for selecting CCA, without difficulty. CCA, common carotid artery.
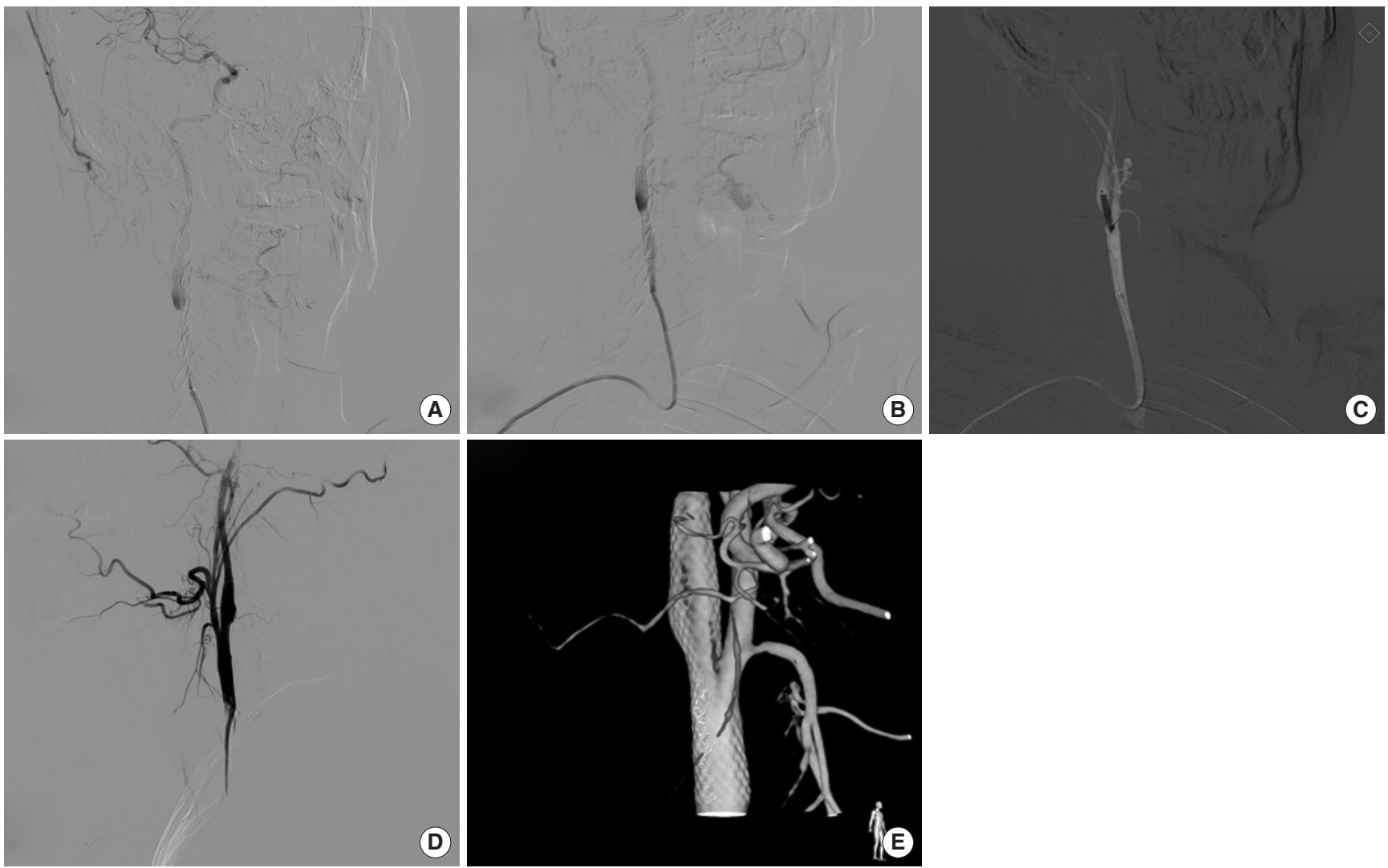

Fig. 6. (A-E) The usual carotid artery stenting technique with distal embolic protection device (FilterWire EZ; Boston Scientific, Marlborough, MA, USA) and Carotid WALLSTENT (Boston Scientific) was done successfully. 

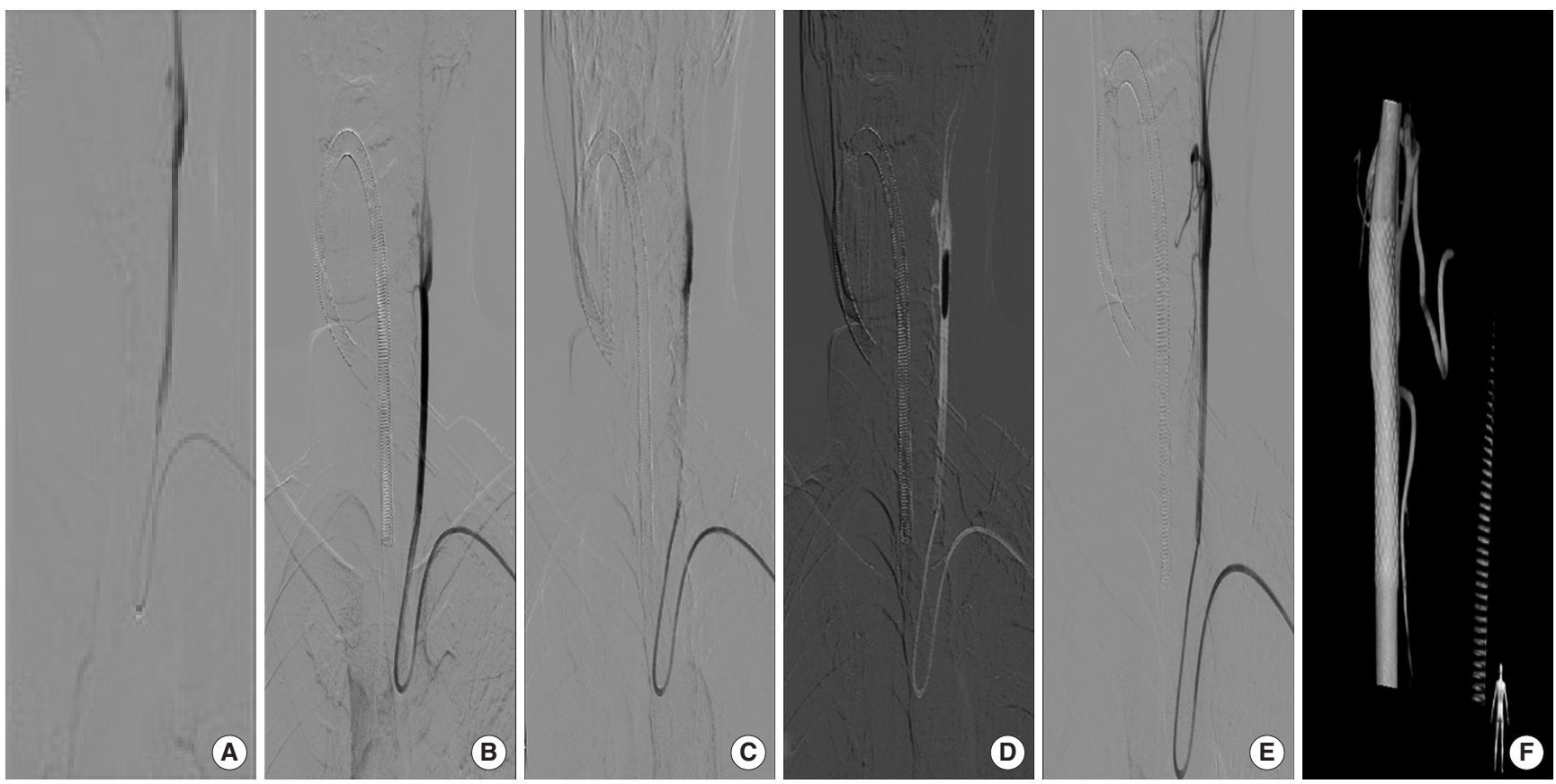

Fig. 7. (A-F) Left carotid stenosis was performed via the left radial artery in the same fashion with the technique described above, uneventfully.

catheter for selecting CCA, without difficulty (Fig. 5). A distal embolic protection device (FilterWire EZ; Boston Scientific, Marlborough, MA, USA) and Carotid WALLSTENT (Boston Scientific) were used successfully (Fig. 6). After the procedure, the patient had an asymptomatic radial artery occlusion (RAO). The left carotid stenosis was planned and we could not use the right radial artery, so the left carotid stenosis was performed via the left radial artery in the same fashion with the technique described above, uneventfully (Fig. 7).

The patient provided written informed consent for the publication of clinical details and images.

\section{DISCUSSION}

CAS is an effective alternative to CEA for the treatment of carotid stenosis. The transfemoral approach is the traditional approach in performing CAS with a high success rate. However, there are situations a transfemoral approach can not be done such as extensive atherosclerotic disease in the aortic arch, iliofemoral occlusive disease, dissection of the thoracic aorta, etc. In such a case, when a transfemoral approach is not available, transbrachial, direct transcervical, or transradial approach can be considered as alternative access [1]. Among them, the transcervical approach is more invasive because it needs a cervical incision to prevent criti- cal access site complications.

Transbrachial approach may also be difficult to be done due to severe complications, including brachial sheath hematoma, compartment syndrome, injury to the medican nerve, and ischemia to the hand [2]. Kiemeneij et al. [3] have reported in their access study comparing transfemoral, transbrachial, and transradial approaches for coronary artery stenting that the transbrachial approach had more major access site complication than the transradial approach. Significant benefit was found in the transradial access having fewer major complications (2.0\% for transfemoral, $2.3 \%$ for transbrachial, $0 \%$ for transradial) and had the same technical success rate as transfemoral and transbrachial access.

Therefore, transradial access can be considered to be the ideal alternative to the traditional transfemoral approach when there are limitations for transfemoral access. The transradial approach was first introduced in 1989 in cardiology and several large interventional cardiology studies have shown the benefit of the transradial approach over the transfemoral approach and now it has been a standard access site in the cardiology field [4]. There are several advantages of transradial access, including the low incidence of access site complications, reduced risk of major bleeding, reduced intensive care unit and hospital length of stay, and more comfortable and better tolerated by patients due to the ability to mobilize earlier post-procedure [5]. However, compared with coronary in- 
Kim SH, et al. - Transradial Carotid Artery Stenting in a Patient with Leriche Syndrome

terventions, transradial access has a technical challenge in the neurointervention field. Selection of the CCA by the radial access will be a difficult task due to the extreme angulation of the common carotid artery at the takeoff point. Despite the advantages of radial access proved in the cardiology field, technical problems made reluctant to switch from femoral to radial access, but there are increasing reports of great success by radial approach for neurointervention procedures [4].

A meta-analysis of seven eligible studies of the radial approach for CAS reported a procedural success rate of 90.8\% [4]. However, this rate of success is lower than the success rate by femoral access. Tokuda et al. [6] reported a success rate of $99.5 \%$ and Shen et al. [7] reported a success rate of $96.2 \%$ for patients with type III aortic arch and $100 \%$ for type I/II arches by femoral access.

Interestingly, Gao et al. [8] included only patients with type III and bovine arch and reported a transradial success rate of $100 \%$ and a transfemoral success rate of $90 \%$. These reports suggest that the overall success rate of femoral access is higher than radial access, but in patients with unfavorable aortic arch morphology for femoral access, such as type III and bovine arches, transradial access can be a better option. Thus, radial access is a complementing rather than competing with the femoral artery approach.

Reports of the complication rate by radial approach are not high. In a systemic review of the literature of transradial approach for neurointervention in 1,342 cases, complications related to the radial approach was classified into minor (asymptomatic and found on routine follow-up or minimally symptomatic, not requiring readmission and intervention) and major (symptomatic and require further intervention). There were two patients $(0.15 \%)$ with a major complication, one patient of symptomatic radial artery occlusion, one patient of large hematoma requiring transfusion, and 37 patients (2.75\%) with minor complications, including 27 patients of asymptomatic RAO, severe radial artery spasm in four patients, and small forearm hematoma in six patients [9]. Allen's test is crucial before transradial approach to prevent major complications of symptomatic RAO. Kuhn et al. [4] reported a distal radial artery access which is a radial approach done distally in the anatomical snuffbox to lower risk of symptomatic RAO. The site of the arteriotomy is distal to the origin of the superficial palmar arch, so even if the access site occludes, the risk of ischemic symptoms will below.

A multicenter prospective randomized study by Ruzsa et al. [10] reported a crossover rate of $10 \%$ in the radial approach and $1.5 \%$ in the femoral approach. There are some contributing factors to the higher crossover rate in the radial access. First, the radial artery has a smaller caliber and arterial spasm occurs frequently making further procedure impossible. So, it is important to inject a spasmolytic 'cocktail' of $200 \mu \mathrm{g}$ nitroglycerine and $2.5 \mathrm{mg}$ of verapamil to prevent vasospasm of the radial artery. Second, inability to cannulate the CCA which has an extreme angulation at its origin. There are some reported techniques to overcome this problem. First, the "telescoping technique"; after engagement of CCA by the Simmons catheter using "pullback and rotate" technique, slowly advance the guiding catheter over the Simmons catheter to CCA. Second, the "retrograde engagement technique"; the guidewire is pushed and creates a loop by the aortic cusp and then engaged in the CCA in a retrograde fashion, and the guiding catheter is advanced over the wire in the same loop fashion inside the CCA.

In our case, we did not use the above techniques. Instead, a guiding catheter, VISTA BRITE TIP (Cordis Corp.) which is used for coronary artery stenting, with a shape of "C" was used. We shaped the tip of the guiding catheter with hot steam to a shape of "U", similar shape to the Simmons catheter to be a shape appropriate for selecting the ostium of CCA and directly selected the CCA with the same technique, "pullback and rotate" used for Simmons catheter. The procedure was simple and there was no difficulty.

In conclusion, CAS is an effective alternative to CEA for the treatment of carotid stenosis. The transfemoral approach is the traditional approach in performing CAS with a high success rate. However, there are situations transfemoral approach can not be done. In such a case, a transradial approach can be a useful alternative approach. We report a case of symptomatic carotid artery stenosis combined with Leriche syndrome which is an aortoiliac occlusive disease, treated by CAS by transradial approach with good result.

\section{REFERENCES}

1. Pinter L, Cagiannos C, Ruzsa Z, Bakoyiannis C, Kolvenbach R. Report on initial experience with transradial access for carotid artery stenting. J Vasc Surg 2007;45:1136-41.

2. Heenan SD, Grubnic S, Buckenham TM, Belli AM. Transbrachial arteriography: indications and complications. Clin Radiol 1996;51:205-9.

3. Kiemeneij F, Laarman GJ, Odekerken D, Slagboom T, van der Wieken R. A randomized comparison of percutaneous transluminal coronary angioplasty by the radial, brachial and femoral approaches: the access study. J Am Coll Cardiol 1997;29:1269-75.

4. Kuhn AL, de Macedo Rodrigues K, Singh J, Massari F, Puri AS. Distal radial access in the anatomical snuffbox for neurointerventions: a feasibility, 
safety, and proof-of-concept study. J Neurointerv Surg 2020;12:798-801.

5. Jaroenngarmsamer T, Bhatia KD, Kortman H, Orru E, Krings T. Procedural success with radial access for carotid artery stenting: systematic review and meta-analysis. J Neurointerv Surg 2020;12:87-93.

6. Tokuda R, Yoshimura S, Uchida K, Yamada K, Satow T, lihara K, et al. Real-world experience of carotid artery stenting in Japan: analysis of 8458 cases from the JR-NET3 nationwide retrospective multi-center registries. Neurol Med Chir (Tokyo) 2019;59:117-25.

7. Shen S, Jiang X, Dong H, Peng M, Wang Z, Che W, et al. Effect of aortic arch type on technical indicators in patients undergoing carotid artery stenting. J Int Med Res 2019;47:682-8.
8. Gao BL, Xu GQ, Wang ZL, Li TX, Wang YF, Liang XD, et al. Transradial stenting for carotid stenosis in patients with bovine type and type III aortic arch: experience in 28 patients. World Neurosurg 2018;111:e661-7.

9. Joshi KC, Beer-Furlan A, Crowley RW, Chen M, Munich SA. Transradial approach for neurointerventions: a systematic review of the literature. J Neurointerv Surg 2020;12:886-92.

10. Ruzsa Z, Nemes B, Pinter L, Berta B, Toth K, Teleki B, et al. A randomised comparison of transradial and transfemoral approach for carotid artery stenting: RADCAR (Radial Access for Carotid Artery Stenting) study. EuroIntervention 2014;10:381-91. 